\title{
Como Avaliar Criticamente Estudos de Coorte em Terapia Intensiva?*
}

\section{How to Critically Assess Intensive Care Cohort Studies?}

\author{
Erica Aranha Suzumura ${ }^{1}$, João Bosco Oliveira ${ }^{1,2}$, Anna Maria Buehler ${ }^{1}$, Mariana Carballo1, Otavio Berwanger ${ }^{1}$
}

\section{RESUMO}

JUSTIFICATIVA E OBJETIVOS: Estudos de coorte são úteis na identificação de fatores de risco e prognósticos, no acompanhamento da história natural de certas doenças e no estudo do impacto de intervenções diagnósticas e terapêuticas. O objetivo deste estudo foi subsidiar o leitor na avaliação crítica de artigos que lançaram mão deste tipo de delineamento de pesquisa.

CONTEÚDO: Na avaliação dos estudos de coorte, é crítico observar a existência de vieses de seleção e informação, a continuidade do seguimento dos sujeitos de pesquisa, o controle dos fatores de confusão, a importância dos resultados e sua aplicabilidade na prática clínica.

CONCLUSÕES: O conhecimento dos fatores que afetam a qualidade dos estudos de coorte permite ao intensivista selecionar as melhores evidências para auxílio na tomada de decisões clínicas.

Unitermos: análise de coorte, delineamento de pesquisa, estudos de coorte, revisão de literatura epidemiológica

1. Instituto de Ensino e Pesquisa do Hospital do Coração (IEP-HCor), São Paulo, SP

2. LIM-56, Faculdade de Medicina da Universidade de São Paulo (FMUSP), São Paulo, SP

*Recebido do Instituto de Ensino e Pesquisa do Hospital do Coração (IEP-HCor), São Paulo, SP

Apresentado em 14 de janeiro de 2008

Aceito para publicação em 22 de janeiro de 2008

Endereço para correspondência:

Dr. Otavio Berwanger

Instituto de Ensino e Pesquisa do Hospital do Coração (IEP-HCor)

Rua Abílio Soares, $250-12^{\circ}$ andar

04005-000 - São Paulo, SP

Fone: (11) 3886-4693 - Fax: (11) 3886-4695

E-mail: otavioberwanger@yahoo.com.br

(C)Associação de Medicina Intensiva Brasileira, 2008

\section{SUMMARY}

BACKGROUND AND OBJECTIVES: Cohort studies are useful to identify risk and prognostic factors, assess disease natural history and verify the impact of diagnostic or therapeutic interventions. This article aims to guide readers on how to critically assess papers using a cohort research design.

CONTENTS: For a critical appraisal of cohort studies, one must observe: the existence of selection and information bias, the continuity of the followup, the control of confounding variables, the significance of the results and its applicability in clinical practice.

CONCLUSIONS: Knowledge of factors which affect the quality of cohort studies allows intensive care professionals to select the best available evidence to guide the decision making process.

Key Words: cohort analysis, cohort studies, epidemiologic research design, literature review

\section{INTRODUÇÃO}

O termo coorte tem origem na área militar, onde correspondia ao agrupamento de 300 a 600 soldados do exército romano. Analogamente, o estudo de coorte consiste no acompanhamento de grupos de pessoas marchando prospectivamente a partir de um determinado momento, sendo expostos a um ou mais fatores de risco'.

Para os intensivistas que desejam adotar a prática da Medicina Baseada em Evidências, é necessário conhecer os diferentes tipos de desenho de estudo e o tipo de questão clínica que cada um deles pode responder.

O objetivo deste estudo foi fornecer subsídios para leitura e avaliação críticas de artigos que utilizam o delineamento de pesquisa tipo coorte, permitindo o adequado julgamento da aplicabilidade dos resultados em situações da prática clínica ${ }^{1}$. 


\section{QUAIS SÃO OS PRINCÍPIOS FUNDAMENTAIS DE UM ESTUDO DE COORTE?}

Em síntese, o estudo de coorte compara experiências de grupos expostos e não expostos a um determinado fator $^{1,2}$. A sua característica principal é o seguimento dos sujeitos de pesquisa no decorrer do tempo para avaliar a ocorrência do desfecho frente ou não a uma exposição ${ }^{2}$, como ilustrado na figura 1.

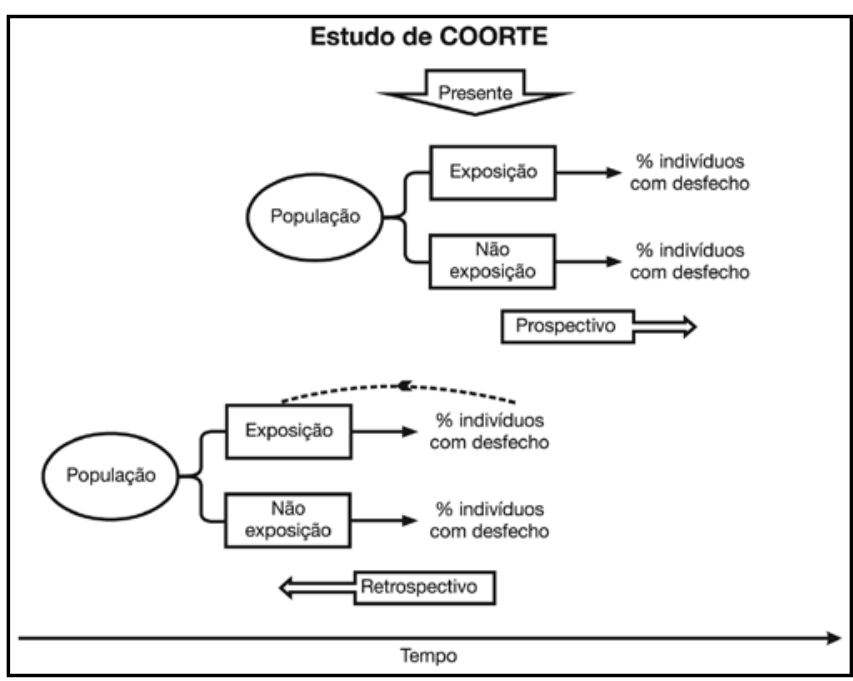

Figura 1 - Delineamento Esquematizado de Estudo de Coorte Prospectivo (parte superior); Delineamento Esquematizado de Estudo de Coorte Retrospectivo (parte inferior).

Há duas formas de realizar um estudo de coorte: selecionam-se os pacientes e o estudo é conduzido no decorrer do tempo, ou pode-se "voltar ao passado" para selecionar os pacientes. Se o investigador redigir o protocolo e as fichas clínicas antes dos dados estarem disponíveis, de forma que os dados sejam coletados de maneira padronizada, este delineamento é chamado de coorte tipo prospectivo ou concorrente. Se o investigador iniciar o estudo quando a exposição, a passagem do tempo e o desfecho já tiverem ocorrido, ou seja, os dados de interesse já existem, ele estará reconstruindo, historicamente, a coorte. Este estudo é chamado de coorte tipo retrospectivo ou não concorrente $e^{2,3}$. Nos dois delineamentos os pacientes selecionados caminham na mesma direção, podendo ter diferentes tempos de arrolamento. Por exemplo, se um investigador pretende determinar a incidência de broncopneumonia em pacientes submetidos à ventilação mecânica invasiva prolongada (mais de 48 horas), pode iniciar sua investigação a partir da data atual ${ }^{4}$. Nesse caso o grupo de pacientes expostos ao fator (ventilação mecânica invasiva por mais de 48h) e o grupo de pacientes não expostos (ventilação mecânica invasiva por menos de $48 \mathrm{~h}$ ) serão acompanhados até o desenvolvimento de broncopneumonia ou até o tempo de encerramento da pesquisa previamente estabelecido pelo investigador, o que ocorrer primeiro. Nessa situação a coorte será prospectiva. Por outro lado, o investigador pode levantar o número de pacientes com broncopneumonia existente em um banco de dados e apurar quantos utilizaram ventilação mecânica invasiva prolongada, ficando expostos ao fator. Nesse caso, tem-se uma coorte retrospectiva.

Existe ainda outra variação de desenho de estudo com três ou mais seguimentos: uma de pacientes não expostos e as demais correspondentes a níveis gradativos de exposição². Empregando o mesmo exemplo, as coortes seriam definidas com base no tempo de ventilação mecânica, em três grupos de pacientes submetidos à ventilação mecânica invasiva: por menos de 48h, por 48-96 horas e mais de 96 horas.

Assim como outros tipos de estudos epidemiológicos observacionais, estudos de coorte podem ser conduzidos com diversas finalidades, incluindo a avaliação de fatores de risco para doenças ou desfechos (p. ex.: presença de insuficiência renal e óbito intra-hospitalar $)^{5}$ ou de sua história natural (p. ex.: evolução da dengue hemorrágica) ${ }^{6}$, estudo do impacto de fatores prognósticos (p. ex.: indicador de necrose miocárdica e mortalidade $)^{7,8}$, intervenções diagnósticas (p. ex.: impacto do uso da proteína-C reativa no diagnóstico de sepse $)^{9}$ e terapêuticas (p. ex.: impacto do tratamento cirúrgico de fraturas de colo de fêmur em idosos sobre a mortalidade $)^{10}$.

\section{COMO AVALIAR CRITICAMENTE ESTUDOS DE CO- ORTE EM TERAPIA INTENSIVA?}

Um bom estudo de coorte devem possuir validades interna e externa. A validade interna avalia se o estudo conseguiu mensurar o que foi proposto pelo delineamento. A validade externa é a capacidade de generalização dos resultados para os pacientes das unidades de terapia intensiva ${ }^{11}$. Aspectos relevantes na avaliação crítica dos estudos de coorte serão descritos a seguir.

\section{O ESTUDO POSSUI VALIDADE INTERNA?}

Para definir se um estudo de coorte possui validade interna, avalia-se a presença de vieses de seleção e informação, a qualidade do seguimento dos sujeitos de 
pesquisa e a presença de fatores de confusão. As características que devem ser levadas em conta durante a avaliação da validade interna de um estudo de coorte estão sumarizadas no quadro 1.

Quadro 1 - Características a serem Avaliadas em um Estudo de Coorte

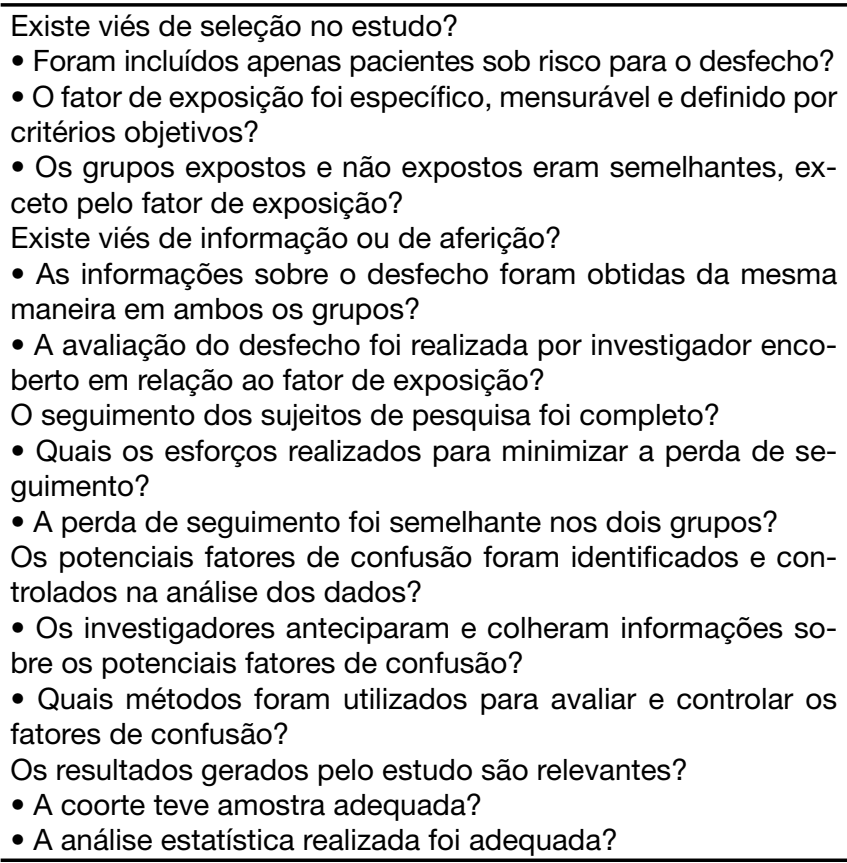

\section{Existe viés de seleção no estudo?}

Foram incluídos apenas indivíduos sob risco para o desfecho?

Todos os participantes (expostos e não expostos) de um estudo de coorte devem estar sob risco de desenvolver o desfecho/doença de interesse. Por exemplo, pacientes histerectomizadas têm risco nulo de desenvolver câncer de útero, portanto, não deveriam ser incluídas em um estudo de coorte cujo desfecho seja câncer neste órgão ${ }^{1}$. Uma má seleção de pacientes contribui para redução na acurácia, ou seja, na capacidade de medir o que o estudo se propõe. Além disso, pacientes sob suspeita de já serem portadores do desfecho em estudo não devem ser incluídos ${ }^{11}$.

O fator de exposição foi específico, mensurável e definido por critérios objetivos?

É necessária a definição a priori da condição de exposto e de não exposto, bem como o grau de exposição. O fator de exposição deve ser específico e mensurável $^{1,2,12}$. Por exemplo, em relação ao uso de fármacos vasoativos (FVAs), as coortes serão 'em uso de FVAs' e 'não uso de FVAs' ${ }^{13}$. Em certos estudos, podem-se até mensurar o grau de exposição ao fator, dependendo do objetivo do estudo, como dosagem de vasopressor infundido, nível de pressão positiva em ventilação mecânica não-invasiva, nível de pressão venosa central, contagem de leucócitos, tempo de protrombina, etc. ${ }^{13-17}$.

Os grupos expostos e não expostos eram semelhantes, exceto pelo fator de exposição?

Sob condições ideais, o grupo não exposto deve ser semelhante ao grupo exposto em todos os aspectos importantes, exceto pela ausência da exposição ao fator sob estudo. Os sujeitos de pesquisa incluídos no estudo devem ter características semelhantes à população alvo, para justificar a generalização externa. Dessa maneira, o grupo não exposto irá revelar a taxa de eventos basal na comunidade ${ }^{1,2,12}$.

\section{Existe viés de informação ou aferição?}

As informações sobre o desfecho foram obtidas da mesma maneira em ambos os grupos?

Depois de determinado tempo de observação, em geral, será verificada a incidência do desfecho nos dois grupos (expostos e não expostos). É preciso, então, que os desfechos sejam bem estabelecidos no planejamento do estudo. As informações sobre os desfechos podem ser oriundas de diversas fontes. Por exemplo, para investigar taxa de mortalidade, geralmente é utilizado o atestado de óbito, porém, para verificar desfechos não-fatais podem ser utilizadas diversas fontes como: prontuário médico, laudo laboratorial, avaliação clínica, registro de alta hospitalar, etc. ${ }^{12,18}$. Se o desfecho de interesse for avaliado por equipamentos, esses devem ter as mesmas especificações e calibragem ${ }^{1,12,19}$. O importante é que os desfechos sejam mensuráveis, comparáveis entre os grupos e sejam definidos pelos mesmos critérios diagnósticos ${ }^{1}$.

A avaliação do desfecho foi realizada por um investigador encoberto em relação ao fator de exposição?

Idealmente, o avaliador dos desfechos não deve ter conhecimento em relação à exposição. A ciência da condição de exposição dos pacientes pode influenciar o julgamento dos desfechos e gerar conclusões errôneas'.

\section{O seguimento dos sujeitos de pesquisa foi completo?}

Quais os esforços realizados para minimizar a perda de seguimento?

A periodicidade do seguimento dos dois grupos de pacientes deve ser previamente definida. Deve ser 
estabelecida a freqüência dos exames médicos para diagnóstico do desfecho em estudo e da coleta de informações pertinentes para se evitar ao máximo as perdas de seguimento. Tais perdas reduzem o poder estatístico dos testes e a precisão das estimativas fazendo com que a questão de pesquisa não seja mais aceita. A melhor maneira de prevenção de perdas de seguimento é a seleção adequada da população do estudo, que deve ser avaliada quanto à possibilidade de continuidade. Além disso, existem outras maneiras de se assegurar o seguimento, como o levantamento de dados relacionados à identificação e destinatário dos sujeitos de pesquisa e pessoas de convívio. A descontinuidade do seguimento é a principal causa de perda de qualidade dos resultados neste tipo de desenho de estudo $20-22$.

\section{A perda de seguimento foi semelhante nos dois grupos?}

Embora a perda de seguimento dos sujeitos de pesquisa diminua o poder e a precisão do estudo, a perda desbalanceada do ponto de vista quantitativo ou qualitativo entre os grupos é ainda mais prejudicial, pois evidencia um evento não aleatório. Se a probabilidade de perda está relacionada à exposição ou ao desfecho, pode haver viés de seguimento. Por exemplo, alguns sujeitos expostos a determinado fator de risco em estudo, que seriam acompanhados após a alta hospitalar, podem evoluir para óbito não comparecendo ao seguimento. A ausência desses pacientes pode sugerir falsamente que o fator em estudo tenha menor relação com o desfecho ${ }^{1,12}$.

\section{Os potenciais fatores de confusão foram identifica- dos e controlados na análise dos dados?}

Os investigadores anteciparam e colheram informações sobre os potenciais fatores de confusão?

Quando uma variável modifica a relação entre o fator de exposição e o desfecho recebe o nome de fator de confusão, sendo normalmente causador da mistura ou sobreposição de efeitos ${ }^{11}$. Por exemplo, em um estudo de coorte sobre o efeito do uso de contraceptivo oral no desenvolvimento de infarto do miocárdio pode-se encontrar o fator de confusão tabagismo. Usuárias de contraceptivo oral também podem ser tabagistas e, neste caso, o cigarro aumenta o risco para infarto do miocárdio. Embora os investigadores esperem avaliar o efeito do contraceptivo oral, estão de fato, avaliando o efeito oculto do cigarro entre mulheres usuárias desse tipo de medicação ${ }^{23}$.
Quais métodos foram utilizados para avaliar e controlar os fatores de confusão?

$O$ viés de confusão, diferentemente dos vieses de seleção e informação, que são irreparáveis, são controláveis desde que informações pertinentes sejam coletadas, visando à homogeneidade entre os grupos do estudo. As estratégias utilizadas para controlar os fatores de confusão incluem:11,12

- Restrição na inclusão de pacientes que apresentem potenciais fatores de confusão;

- Pareamento dos sujeitos incluídos nos grupos expostos e não expostos (ambos os grupos contém pacientes com fatores de confusão na mesma proporção);

- Análise em subgrupos, que pode ser considerada uma forma post hoc de restrição, feita durante a análise dos resultados por intermédio de métodos estatísticos;

- Técnicas de análises múltiplas, onde modelos estatísticos examinam o potencial efeito de uma variável enquanto simultaneamente controlam o efeito de outros fatores.

A realização de análises em subgrupos (estratificadas) e análises múltiplas exige cautela, pois, a amostra inicialmente calculada pode não suportar tantas comparações. Aconselha-se manter no mínimo 10 eventos (desfechos) para cada fator acrescentado numa análise. Uma descrição mais detalhada destas técnicas foge ao escopo desta publicação.

\section{Os resultados obtidos pelo estudo são relevantes?}

A coorte teve amostra adequada?

A amostra normalmente é planejada para responder a questão de pesquisa: identificar associação entre o desfecho e o fator em estudo. Assim, os pacientes serão classificados como desfecho positivo (doentes) ou desfecho negativo (não doentes) na presença do fator em questão. O ideal é que todos os pacientes sejam classificados corretamente. Entretanto, às vezes erros são cometidos, ou seja, um paciente doente é dito como não doente (erro tipo I) e um não doente é avaliado como doente (erro tipo II). Assim, um erro tipo I é controlado no delineamento do estudo pelo nível de significância adotado $(\alpha)$, sendo sempre menor que o erro tipo II ( $\beta$ ). O erro tipo II é controlado indiretamente pelo poder do teste, uma vez que o poder é representado por $1-\beta$. O poder do teste representa a capacidade do teste estatístico evidenciar a questão de pesquisa.

Dessa forma, um estudo deve ter tamanho suficiente para evitar os dois tipos de erros: Erro tipo I (afirmar 
que o fator de risco está associado com o desfecho e de fato não está) e Erro tipo II (afirmar que o fator de risco não está associado com o desfecho e de fato está). A quantidade de sujeitos incluídos em um estudo de coorte em UTI depende dos seguintes itens: 1) incidência da doença nos dois grupos, 2) estimativa de risco relativo associado à exposição, 3) nível de significância desejado, normalmente $5 \%$, 4) poder do estudo, normalmente $80 \%$. Ao número mínimo de pacientes calculado em cada grupo, acrescentam-se $10 \%$ ou $20 \%$ para suprir eventuais perdas ${ }^{2,12}$. A seguir, estimativas de tamanho de amostra segundo proporção fictícia de expostos à sepse sobre mortalidade em UTI (Tabela 1).

\section{A análise estatística realizada foi adequada?}

A maioria dos estudos de coorte tem como objetivo primário avaliar associação entre a incidência do desfecho em pacientes expostos e não expostos, sendo normalmente utilizado o teste Qui-quadrado quando não há fator de confusão.

Se a associação for significativa, verifica-se o grau de associação através do cálculo do risco relativo (RR), que é a razão entre a incidência nos expostos (probabilidade do indivíduo ficar doente dado que foi exposto ao fator de estudo) pela incidência nos não expostos (probabilidade de ficar doente dado que não foi exposto). O RR representa quantas vezes mais o paciente exposto está sujeito a desenvolver a doença em relação ao não exposto. Quanto maior o RR, maior será a convicção de que o fator em estudo está associado à doença em questão. Se o RR for igual a 1, a incidência entre os expostos e não expostos é igual, ou seja, o fator de exposição em estudo não tem relação com a doença.

A significância do RR é calculada pelo intervalo de confiança (IC). Se o IC contiver o valor 1 , significa que o fator de exposição parece não acrescentar risco para a doença. Se o IC não contiver o valor 1 , o fator de exposição acrescenta risco para a doença. Uma vez evidenciada a presença da associação, calcula-se o risco atribuível na população (RAP).

O RAP é a diferença entre os coeficientes de incidência entre os dois grupos (este coeficiente considera o fator "tempo de seguimento" dos sujeitos de pesquisa). O RAP fornece uma estimativa do impacto que as medidas preventivas teriam ao eliminar ou diminuir o fator de exposição².

\section{O ESTUDO POSSUI VALIDADE EXTERNA?}

Se os resultados forem relevantes e todas as possíveis causas de vieses forem descartadas, então, os dados gerados pela pesquisa devem ser avaliados em relação à validade externa, ou seja, a aplicabilidade clínica ${ }^{11}$. A generalização dos resultados de um estudo de coorte em terapia intensiva depende da semelhança entre características basais dos pacientes incluídos no estudo e os pacientes críticos. Além disso, os pacientes internados devem apresentam o fator em estudo definido pelos mesmos parâmetros adotados no artigo científico. Se seus pacientes preencherem os critérios de inclusão e exclusão estabelecidos pelo estudo de coorte, não há muita discussão quanto à aplicabilidade, ou seja, pacientes expostos àqueles fatores serão mais suscetíveis ao desfecho, necessitando de maiores cuidados.

\section{CONCLUSÃO}

Os estudos de coorte são ferramentas importantes para o conhecimento dos fatores de risco associados com inúmeras doenças que necessitam de cuidados intensivos, das taxas de incidência de desfechos importantes e da evolução natural de pacientes críticos em UTI. Entretanto, é necessário que os resultados sejam relevantes e provenham de estudos que tenham validade interna. O conhecimento dos fatores que agregam qualidade aos estudos de coorte permite o julgamento crítico da literatura disponível, o que pode auxiliar a tomada de decisões na prática da terapia intensiva.

Tabela 1 - Estimativas de Tamanho de Amostra segundo Proporção de Expostos sobre Mortalidade em UTI

\begin{tabular}{|c|c|c|c|c|c|c|}
\hline Fator de Exposição & Proporção de Expostos & $\mathrm{RR}$ & Poder & Nível de Significância & Amostra (por grupo) & Amostra Tota \\
\hline Sepse & $25 \%$ & 1,5 & $80 \%$ & $5 \%$ & 371 & 742 \\
\hline Sepse & $25 \%$ & 1,5 & $80 \%$ & $1 \%$ & 554 & 1108 \\
\hline Sepse & $25 \%$ & 1,5 & $90 \%$ & $5 \%$ & 497 & 994 \\
\hline Sepse & $25 \%$ & 1,5 & $90 \%$ & $1 \%$ & 705 & 1410 \\
\hline Choque séptico & $40 \%$ & 2,0 & $80 \%$ & $5 \%$ & 81 & 162 \\
\hline Choque séptico & $40 \%$ & 2,0 & $80 \%$ & $1 \%$ & 121 & 242 \\
\hline Choque séptico & $40 \%$ & 2,0 & $90 \%$ & $5 \%$ & 108 & 216 \\
\hline Choque séptico & $40 \%$ & 2,0 & $90 \%$ & $1 \%$ & 154 & 308 \\
\hline
\end{tabular}




\section{REFERÊNCIAS}

01. Grimes DA, Schulz KF - Cohort studies: marching towards outcomes. Lancet, 2002;359:341-345.

02. Haddad N - Delineamento de Estudos Analíticos, em: Haddad N Metodologia de Estudos em Ciências da Saúde: como Planejar, Analisar e Apresentar um Trabalho Científico. São Paulo: Editora Roca, 2004;39-70.

03. Coeli CM, Faerstein E - Estudo de Coorte, em: Medronho RA, Carvalho DM, Bloch KV et al - Epidemiologia. São Paulo: Atheneu, 2003;161-173.

04. Carrilho CM, Grion CM, Bonametti AM et al - Multivariate analysis of the factors associated with the risk of pneumonia in intensive care units. Braz J Infect Dis, 2007;11:339-344.

05. Mayr VD, Dunser MW, Greil V et al - Causes of death and determinants of outcome in critically ill patients. Crit Care, 2006;10:R154.

06. Salgado DM, Rodríguez JA, Garzón M et al - Clinical and epidemiological characterization of dengue haemorrhagic fever in Neiva, Colombia, 2004. Rev Salud Publica (Bogota), 2007;9:53-63.

07. Kim LJ, Martinez EA, Faraday $\mathrm{N}$ et al - Cardiac troponin I predicts short-term mortality in vascular surgery patients. Circulation, 2002;106:2366-2371.

08. Filipovic $M$, Jeger $\mathrm{R}$, Probst $\mathrm{C}$ et al - Heart rate variability and cardiac troponin I are incremental and independent predictors of one-year allcause mortality after major noncardiac surgery in patients at risk of coronary artery disease. J Am Coll Cardiol, 2003;42:1767-1776.

09. Ceccon ME, Vaz FA, Diniz EM et al - Interleukins 6 and C-reactive protein for the diagnosis of late onset sepsis in the newborn infant. Rev Assoc Med Bras, 2006;52:79-85.

10. Marker DR, Seyler TM, Jinnah $\mathrm{RH}$ et al - Femoral neck fractures after metal-on-metal total hip resurfacing: a prospective cohort study. J Arthroplasty, 2007;22:(7 Suppl3):66-71.

11. Grimes DA, Schulz KF - Bias and causal associations in observational research. Lancet, 2002;359:(9302):248-252.
12. White E, Hunt JR, Casso D - Exposure measurement in cohort studies: the challenges of prospective data collection. Epidemiol Rev, 1998;20:43-56.

13. Arnold LM, Crouch MA, Carroll NV et al - Outcomes associated with vasoactive therapy in patients with acute decompensated heart failure. Pharmacotherapy, 2006;26:1078-1085.

14. Sinuff $\mathrm{T}$, Cook $\mathrm{D}$, Randall $\mathrm{J}$ et al - Noninvasive positive-pressure ventilation: a utilization review of use in a teaching hospital. CMAJ, 2000;163:969-973.

15. George IA, John $G$, John $P$ et al - An evaluation of the role of noninvasive positive pressure ventilation in the management of acute respiratory failure in a developing country. Indian J Med Sci, 2007;61:495-504.

16. Weisfelt $M$, van de Beek $D$, Spanjaard $L$ et al - Attenuated cerebrospinal fluid leukocyte count and sepsis in adults with pneumococcal meningitis: a prospective cohort study. BMC Infect Dis, 2006;6:149.

17. Brass LM, Lichtman JH, Wang Y et al - Intracranial hemorrhage associated with thrombolytic therapy for elderly patients with acute myocardial infarction: results from the Cooperative Cardiovascular Project. Stroke, 2000;31:1802-1811.

18. Tager IB - Outcomes in cohort studies. Epidemiol Rev, 1998;20:15-28

19. Whitney CW, Lind BK, Wahl PW - Quality assurance and quality control in longitudinal studies. Epidemiol Rev, 1998;20:71-80.

20. Hunt JR, White E - Retaining and tracking cohort study members. Epidemiol Rev, 1998;20:57-70.

21. Austin MA, Berreyesa S, Elliott JL et al - Methods for determining long-term survival in a population based study. Am J Epidemiol, 1979;110:747-752.

22. Desmond DP, Maddux JF, Johnson TH et al - Obtaining follow-up interviews for treatment evaluation. J Subst Abuse Treat, 1995;12:95-102.

23. Owen-Smith V, Hannaford PC, Warskyj M et al - Effects of changes in smoking status on risk estimates for myocardial infarction among women recruited for the Royal College of General Practitioners' Oral Contraception Study in the UK. J Epidemiol Community Health, 1998;52:420-424. 